\title{
Adenoviral hepatitis in an immunocompetent child: Case report
}

Volume 7 Issue 3 - 2017

Keywords: adenovirus, hepatitis, polymerase chain reaction

\section{Introduction}

Acute liver failure is considered when severe and sudden liver cell dysfunction leading to hepatic encephalopathy and coagulopathy in previously healthy persons occurs. A critical degree of liver cell death, not adequately decompensated by hepatocellular regenerative action, is crucial for the development of acute liver failure. Interface between two dominant pathological pathways is seen as the triggering factor: apoptosis and necrosis. The etiology of acute liver failure affects the dominant pathway. Liver cell death signaling pathways is controlled by an increasingly number of proinflammatory and vasoactive cytokines, transcription factors, tyrosine kinases, adapter molecules, by stimulation and depression of these factors. ${ }^{1}$ However, if no enough necrosis and apoptosis occurs, liver failure may be salvaged. Hence, hepatitis may be the result.

\section{Case report}

We report the case of a six year old girl presenting for management of jaundice. The patient was born premature, had a history of Necrotizing enterocolitis, with normal development, and was the first child of unrelated, healthy parents. There was no history of previous liver disease nor exposure to a toxic agent or administration of medications known to cause hepatitis.

One week prior to presentation patient started having upper respiratory tract symptoms and was given amoxicillin clavulonic acid for tonsillitis with no documented streptococcal infection. Three days after starting the amoxiclave patient started having vomiting and diarrhea with abdominal pain and decrease enteral feeding. Jaundiced started and persisted then worsened until the day of presentation. Upon presentation, patient had no more vomiting, no more diarrhea and she was afebrile. Her only complaint was jaundice. No somnolence nor bleeding were reported. To note no change nor in urine color nor in stools color were noted.

On physical examination, her weight was $20 \mathrm{~kg}$ (50 percentile) and height $100 \mathrm{~cm}$ (50 percentile). She was jaundiced. Her abdomen was soft and there was no hepatosplenomegaly or ascites. The remainder of her physical examination was normal. Patient was afebrile.

Labs showed direct hyperbilirubinemia with a direct of $12.7 \mathrm{mg}$ / $\mathrm{dl}$ and a total of $14.4 \mathrm{mg} / \mathrm{dl}$, glucose of $85 \mathrm{mg} / \mathrm{dl} \mathrm{GGT}$ of $334 \mathrm{U} / \mathrm{L}$, ALK 537 U/L, SGPT 370 U/L, and SGOT 1108 U/L. Tests for liver function were normal. Patient had a normal coagulation profile.

Abdominalultrasoundshowednormalliver, nohepatosplenomegaly, a normal common bile duct, a thickened gallbladder, a non-distended gallbladder, however few lymph node in hepatic hilum with $7 \mathrm{~mm}$ the largest were seen. Pancreas, spleen and kidney were normal.

Hepatitis A to E viruses, and those in which liver involvement may occur as part of disseminated infection, such as EpsteinBarr virus

\author{
Pia Kiwan, Dany Al Hamod \\ Department of Pediatrics, Saint George Hospital University \\ Medical Center, University of Balamand, Lebanon
}

Correspondence: Dany Al Hamod, Department of Pediatrics, Saint George University Hospital, University of Balamand, Beirut, Lebanon,Tel 96I-368-3209, Email dany_alhamod@hotmail.com

Received: October 25, 2017 | Published: November 16, 2017

(EBV), cytomegalovirus (CMV), varicella zoster virus and herpes simplex virus (HSV) were ordered. To note, patient was immune to hepatitis A and B. Hepatitis workup was all negative. EBV, CMV and HSV panels were also negative.

A work up was done for probable noninfectious causes of hepatitis. ANA, anti-smooth muscle antibody, and anti-liver kidney microsomal antibody assays were negative; serum ceruloplasmin and alphafetoprotein levels were within normal limits. Wilson disease was ruled out. Repeated labs the next day, only after hydration, showed a decrease in the liver enzymes: SGOT dropped to $683 \mathrm{U} / \mathrm{L}$, SGPT to $255 \mathrm{U} / \mathrm{L}, \mathrm{GGT}$ to $283 \mathrm{U} / \mathrm{L}, \mathrm{ALK}$ to $537 \mathrm{U} / \mathrm{L}$.

Ursodeoxycholic acid was started.

Autoimmune hepatitis was ruled out with negative ANA anti Ds DNA and negative immune liver profile.

Onset of hepatic injury in the present case appeared to occur concomitantly or just after respiratory tract infection and the watery diarrhea. Therefore, an infectious disease causing acute liver injury was highly possible. Lack of serologic evidence of other etiologic factors causing viral hepatitis, diarrhea and intestinal invagination led us to search for adenovirus in the stool and blood (adenovirus serology and PCR). To note, it is known that the presence of the virus in the stool does not indicate clinical adenovirus infection because these viruses may be excreted chronically and asymptomatically. But, in these instances, discovery of a coincident increase in antibody is helpful in the diagnosis. ${ }^{2}$. So adenoviral PCR was ordered. Results were positive. Since there was positive adenovirus IgM and IgG antibody, and positive blood PCR, it was conceivable that our patient had primary adenovirus infection.

Samples were then subjected to adenovirus nucleic acid detection by PCR. Results came back positive in blood and stools.

Our patient was given supportive care. Gradually, her LFTs returned to baseline. Ten days after discharge patient had repeat of her laboratory exams and these were the following: PT INR 1.05, PTT 40 seconds, Glucose $83 \mathrm{mg} / \mathrm{dl}$. These were normal however the 
liver enzymes were still elevated: Total bilirubin $2.9 \mathrm{mg} / \mathrm{dl}$, Direct bilirubin2.4 mg/dl, SGOT: $265 \mathrm{U} / \mathrm{L}$, SGPT: $166 \mathrm{U} / \mathrm{L}$, GGT: 141 U/L and ALK: 338U/L. Repeated labs within one month showed normalization: SGOT 24 U/L, SGPT 17 U/L, GGT 18U/L, ALK of $177 \mathrm{U} / \mathrm{L}$. Total bilirubin $0.6 \mathrm{mg} / \mathrm{dl}$, and a direct bilirubin of $0.3 \mathrm{mg} / \mathrm{dl}$. Glucose $88 \mathrm{mg} / \mathrm{dl}$. PTT 35 seconds and PT INR of 1.18.

\section{Discussion}

Although rare, adenovirus infection should be considered in the differential diagnosis of acute hepatitis in immunocompetent children. In healthy children, adenoviral infection causes a benign, self-limited illness, and any virus that can cause acute hepatitis may potentially give rise to acute liver failure. The clinical course of adenovirus infection among healthy children can be complicated by severe or fatal pneumonia, myocarditis, and hepatitis. ${ }^{3}$

Adenoviruses are more and more documented as contributors to morbidity and mortality among immunodeficient patients, for example acute leukemia patients, bowel transplant patients and stem cell and solid-organ transplant recipients. ${ }^{4}$

Adenovirus accounts for $5 \%$ to $10 \%$ of upper and lower respiratory tract infections in infants and children and generally causes a benign, self-limited illness. Transmission is by respiratory and fecal oral routes. ${ }^{5}$

Human adenoviruses (HAdVs), which have been suggested as one of the causes of acute viral hepatitis in immunocompromised patients, consist of nonenveloped, double-stranded DNA and belong to the family Adenoviridae, genus Mastadenovirus. It includes 51 distinct serotypes that cause disease in humans. The 51 recognized serotypes of human adenoviruses have been placed in 7 human adenovirus species, A-G. ${ }^{3}$ In healthy children, adenoviral infection causes a benign, self-limited illness. Acute liver failure from adenovirus is rare, and is described especially in immunocompromised patients, in whom it is frequently fatal. Depending on the species, these viruses may infect respiratory, conjunctival, gastrointestinal, and genitourinary sites. To note, fulminant hepatitis is a rare complication of adenoviral infection. ${ }^{6}$

Serotypes $1,2,3$, and 5 cause mainly respiratory illness, and serotypes 40 and 41 cause mainly gastroenteritis. The cases of acute respiratory disease are most frequently seen with a strain of HAdV-B, HAdV-7. More virulent types (particularly type 7) can cause respiratory failure, shock and hepatitis in immunocompetent children (Table 1). At Texas Children's Hospital, a retrospective review of adenovirus infections during 1990-1996 was performed to assess the epidemiology, clinical course, management, and result of disseminated adenovirus disease (DAD) in children. DAD with multiorgan involvement occurred in $11(2.5 \%)$ of 440 adenovirusinfected patients. Six (54\%) of the 11 were immunocompromised and $5(45 \%)$ were immunocompetent. Mortality was $83 \%$ among the immunodeficient, $60 \%$ in the immunocompetent, and $73 \%$ overall.

DAD was more common in immunocompromised children. ${ }^{7}$

Table I Infections associated with adenovirus subgroup and serotype

\begin{tabular}{lll}
\hline Subgroup & Serotype(s) & Major site(s) of infection \\
\hline A & $I 2, I 8,3 I$ & Respiratory tract, urinary tract, GI tract \\
B & $3,7, I 6,2 I, I I, I 4,34,35$ & Respiratory tract, eye, urinary tract, GI tract \\
C & $I, 2,5,6$ & Respiratory tract, urinary tract, GI tract \\
$D$ & $8-10, I 3, I 5, I 7, I 9,20,22-30,32,33,36-39,42-49$ & Eye, GI tract \\
E & 4 & Eye, respiratory tract \\
F & $40,4 I$ & Gl tract \\
\hline
\end{tabular}

Note: From ${ }^{1-3}$. Gl, gastrointestinal.

Rocholl et al. ${ }^{8}$ reported that disseminated adenoviral disease occurred at a younger age in immunocompetent children. They also described in immunocompromised patients cases with fulminant hepatic failure, colitis, pancreatitis, pneumonitis, nephritis, meningoencephalitis and encephalopathy due to adenovirus infection. In the Kuala Lumpur Police Training Centre, an outbreak of ARD, caused by HAdV-7, started in early March 2011. The police trainees had signs and symptoms of acute respiratory disease such as fever, cough, and loss of appetite. Although most adenovirus infections are self-limited, approximately 100 trainees were hospitalized and 5 were admitted to the intensive care unit. Three of these 5 trainees died. Human adenovirus type 7 was identified as the etiologic agent. ${ }^{7}$

Several methods are used to detect adenovirus infection depending on the site and severity of infection. Adenovirus can be shown by immunohistology or characteristic pathologic changes including intranuclear inclusion bodies in biopsy material, isolation of virus by culture or PCR, or demonstration of an increase in antibody titers. ${ }^{2}$ However, the histological examination of a postmortem liver biopsy specimen was reported as massive hepatic necrosis without specific inclusion bodies for adenovirus thus the importance of PCR on the biopsy material.

Several authors have commented on the difficulty of confirming the adenoviral infection by histopathology. Flomenberg et al.. ${ }^{4}$ reported that only three of eight bone marrow patients with positive adenoviral stool cultures had diagnostic viral histopathology. Similarly, Parizhkaya et al. ${ }^{10}$ reported only three of 70 small bowel transplant recipients diagnosed with adenovirus enteritis had characteristic epithelial changes in small bowel biopsy. Thus, histopathology is not a sensitive method in diagnosing adenoviral infection. ${ }^{4}$

Isolation of adenovirus from possible infectious sites (urine, stool, respiratory secretion, and cerebrospinal fluid) is not always fruitful. Negative cultures for adenovirus from body fluids do not eliminate adenovirus infection, because of the limited sensitivity. On the other hand, positive viral cultures do not provide evidence of invasive or disseminated disease due to shedding of epithelial cells harboring adenovirus. $^{4}$

Blood PCR test is sensitive in the case of acute liver failure or hepatitis. In addition, PCR is a very useful method in the detection of adenovirus in biopsy tissues. ${ }^{1}$. However, secondary to massive hepatic necrosis in liver failure patients, liver adenovirus PCR tested in the liver tissue can be found negative. Blood PCR test may be more sensitive than liver PCR test in the case of acute liver failure with massive hepatic necrosis.

Liver failure arises from an imbalance between liver cell death and regeneration. Liver cell death occurs through complex cellular 
interactions and is mediated by inflammatory, immunological and chemical components. Whether adenovirus infection can trigger immune-mediated acute hepatitis must be considered. In conclusion, we recommend that adenovirus infection should be included in the differential diagnosis of hepatitis seen in childhood. ${ }^{11-19}$ In conclusion, although rare, we recommend that adenovirus infection should be included in the differential diagnosis of hepatitis seen in immunocompetent children.

\section{Acknowledgments}

We thank the administration of The university Hospital of Saint George Beirut Lebanon for permission to publish this article.

\section{Conflicts of interest}

None.

\section{References}

1. Horwitz MS. Adenoviruses. In:Knipe DM \& Howley PM, editors. Fields' virology. 4th edn, Lippincott Williams \&amp; Wilkinsp, Philadelphia, USA, 2001. pp. 2301-2326.

2. Yamadera S, Yamashita K, Akatsuka M, et al. Adenovirus type 7 outbreaks in Japan in 1998. Jpn J Infect Dis. 2000;53:22-23.

3. Amdani S, Sathyaprasad A, Badheka A, et al. Adenoviral Hepatitis in an Immunocompetent Child. 2012.

4. Flomenberg P, Babbitt J, Drobyski WR, et al. Increasing incidence of adenovirus disease in bone marrow transplant recipients. J Infect Dis. 1994;169(4):775-781.

5. Schmitz H, Wigand R, Heinrich W. Worldwide epidemiology of human adenovirus infections. Am J Epidemiol. 1983;117(4):455-466.

6. Aspasia Soultati. SP Dourakis. Pathogenesis of Fulminant Hepatic Failure. Annals of Gastroenterology. 2006;19(4):313-324.

7. Munoz FM, Piedra PA, Demler GJ. Disseminated adenovirus disease in immunocompromised and immunocompetent children. Clinical Infectious Diseases. 1998;27(5):1194-1200.
8. Rocholl C, Gerber K, Daly J, et al. Adenoviral infections in children: the impact of rapid diagnosis. Pediatrics. 2004;113(1 Pt 1):51-55.

9. Yusof MA, Rashid TR, Thayan R, et al. Human Adenovirus Type 7 Outbreak in Police Training Center, Malaysia, 2011. Emerg Infect Dis. 2011;18(5):852-854.

10. Parizhskaya M, Walpusk J, Mazariegos G, et al. Enteric adenovirus infection in pediatric small bowel transplant recipients. Pediatr Dev Pathol. 2001;4(2):122-128.

11. Seo YM, Hwang-Bo S, Kim SK, et al. Fatal systemic adenoviral infection superimposed on pulmonary mucormycosis in a child with acute leukemia: A case report. Medicine (Baltimore). 2016;95(40):e5054.

12. Ozbay Hoşnut F, Canan O, Ozçay F, et al. Adenovirus infection as possible cause of acute liver failure in a healthy child:a case report. Turk J Gastroenterol. 2008;19(4):281-283.

13. Ison MG. Adenovirus Infections in Transplant Recipients. Clinical Infectious Diseases. 2006;43(3):331-339.

14. Williams R, Riordan SM. Acute liver failure: established and putative hepatitis viruses and therapeutic implications. $J$ Gastroenterol Hepatol. 2000;15(Suppl):G17-G25.

15. Mitchell DK, Pickering LK. Gastroenteritis. In: Katz SL, et al. editors. Krugman's infectious diseases of children. 10th edn, Mosby Press, St. Louis, USA. 1998. pp. 116-139.

16. McIntosh K. Adenoviruses. In: Behrman RE, et al. editors. Nelson Textbook of Pediatrics. 16th edn, WB Saunders Co, Philadelphia, USA. 2000. pp. 994-995.

17. Hough R, Chetwood A, Sinfield R, et al. Fatal adenovirus hepatitis during standard chemotherapy for childhood acute lymphoblastic leukemia. J Pediatr Hematol Oncol. 2005;27(2):67-72.

18. Seidemann K, Heim A, Pfister ED, et al. Monitoring of adenovirus infection in pediatric transplant recipients by quantitative PCR:report of six cases and review of the literature. Am J Transplant. 2004;4(12):2102-2108.

19. Peled N, Nakar C, Huberman H, et al. Adenovirus infection in hospitalized immunocompetent children. Clin Pediatr (Phila). 2004;43(3):223-229. 